\title{
3dRS, a Web-Based Tool to Share Interactive Representations of 3D Biomolecular Structures and Molecular Dynamics Trajectories
}

\author{
Genís Bayarri ${ }^{1}$, Adam Hospital ${ }^{1 *}$ and Modesto Orozco ${ }^{1,2 *}$ \\ ${ }^{1}$ Institute for Research in Biomedicine (IRB Barcelona), The Barcelona Institute of Science and Technology (BIST), Barcelona, \\ Spain, ${ }^{2}$ Departament de Bioquímica i Biomedicina, Facultat de Biologia, Universitat de Barcelona, Barcelona, Spain
}

OPEN ACCESS

Edited by:

Brian Jiménez-García,

Utrecht University, Netherlands

Reviewed by:

Ezgi Karaca

Dokuz Eylul University, Turkey

Sjoerd Jacob De Vries,

Institut National de la Santé et de la

Recherche Médicale, France

*Correspondence:

Adam Hospital

adam.hospita/@irbbarcelona.org

Modesto Orozco

modesto.orozco@irbbarcelona.org

Specialty section:

This article was submitted to Biological Modeling and Simulation,

a section of the journal

Frontiers in Molecular Biosciences

Received: 16 June 2021

Accepted: 03 August 2021

Published: 13 August 2021

Citation:

Bayarri G, Hospital A and Orozco M (2021) 3dRS, a Web-Based Tool to Share Interactive Representations of

3D Biomolecular Structures and Molecular Dynamics Trajectories.

Front. Mol. Biosci. 8:726232.

doi: $10.3389 /$ /fmolb.2021.726232
3D Representation Sharing (3dRS) is a web-based tool designed to share biomolecular structure representations, including 4D ensembles derived from Molecular Dynamics (MD) trajectories. The server offers a team working in different locations a single URL to share and discuss structural data in an interactive fashion, with the possibility to use it as a live figure for scientific papers. The web tool allows an easy upload of structures and trajectories in different formats. The 3D representation, powered by NGL viewer, offers an interactive display with smooth visualization in modern web browsers. Multiple structures can be loaded and superposed in the same scene. 1D sequences from the loaded structures are presented and linked to the 3D representation. Multiple, pre-defined 3D molecular representations are available. The powerful NGL selection syntax allows the definition of molecular regions that can be then displayed using different representations. Important descriptors such as distances or interactions can be easily added into the representation. Trajectory frames can be explored using a common video player control panel. Trajectories are efficiently stored and transferred to the NGL viewer thanks to an MDsrv-based data streaming. The server design offers all functionalities in one single web page, with a curated user experience, involving a minimum learning curve. Extended documentation is available, including a gallery with a collection of scenes. The server requires no registration and is available at $\mathrm{https} / / / \mathrm{mmb}$.irbbarcelona.org/3dRS.

Keywords: 3D representation, biomolecular structures, data sharing, molecular dynamics, interactive figures, FAIR

\section{INTRODUCTION}

Since Max Perutz and colleagues solved the first 3D structure of Hemoglobin using X-ray crystallography in 1960 (Perutz et al., 1960), macromolecular structure 3D representations have become classic elements in biomolecular scientific journals and scientific discussions. The constant exponential growth in the number of experimentally solved structures deposited in the Protein Data Bank database (Berman et al., 2000) (PDB) and the spectacular increase in the reliability of Molecular

\footnotetext{
Abbreviations: PDB, Protein Data Bank; MD, Molecular Dynamics; VM, Virtual Machine; SPA, Single Page Application; VS, Virtual Screening; CPU, Central Processing Unit; GPU, Graphics Processing Unit; WebGL, Web Graphics Library; PDF, Portable Document Format; API, Application Programming Language; HBP, Human Brain Project;
} 
Dynamics (MD) simulations (Hospital et al., 2015), Virtual Screening (VS) techniques (Walters and Wang, 2020) or protein structure predictions (Senior et al., 2020; Zhu et al., 2021) generates the need to deal in an efficient way with structures (3D) and structural ensembles (4D models, with time as a $4^{\text {th }}$ dimension). The still dominant use of flat figures or predetermined videos is hampering discussion and full exploitation of the power of structural data.

Informatics technology has evolved even faster than structural biology. Thus, current network bandwidths allow streaming of heavy videos at home, Central and Graphics Processing Units (CPUs, GPUs) are powerful enough to work with extremely demanding tasks, even in portable and small devices, and graphic software has improved dramatically their performance for realistic representations of the structural models. Some of these technologies (such as WebGL) have been already used for the representation of 3D structures in a common web browser (Rego and Koes, 2015; Bekker et al., 2016; Sehnal et al., 2017; Shi et al., 2017; Perkel, 2018; Rose et al., 2018; Hildebrand et al., 2019; Sehnal et al., 2021) using native support for GPU acceleration. Web applications and online platforms have already integrated these tools, starting with the PDB databank, which offers the possibility to represent the uploaded structures with NGL (Rose et al., 2018), Mol ${ }^{\star}$ (Sehnal et al., 2021) or JSMol. However, representation of MD simulation data is not as common. Although tools to automate the generation of MD videos exist (Johnson et al., 2011; Andrei et al., 2012; Wieczór et al., 2020), they lack interactivity. In terms of web-based interactive visualizers, only some of them are able to read MD trajectory data (e.g. JSMol, NGL), and are restricted to a few trajectory formats. A small number of servers are integrating MD data visualization with different degrees of interactivity (Meyer et al., 2010; Hospital et al., 2016; Rodríguez-Espigares et al., 2020; Zivanovic et al., 2020). Luckily, tools such as MDsrv (Tiemann et al., 2017) and HTMol (Carrillo-Tripp et al., 2018) came recently into play to facilitate this task, allowing streaming and visualization of MD trajectories. MDsrv accepts many different trajectory formats, thanks to the help of MDTraj (McGibbon et al., 2015) and MDAnalysis (Michaud-Agrawal et al., 2011) software packages.

However, despite all these technological advances, the practical use of $3 \mathrm{D} / 4 \mathrm{D}$ structural images still implies visualization in a single computer, which means that interactivity with the structure is limited to a handful of scientists located in a particular laboratory. The inability to interact with $3 \mathrm{D} / 4 \mathrm{D}$ structures is especially annoying when dealing with published material. Thus, conference proceedings and journals provide electronic copies of the papers, including pictures or in some cases videos of the structures. However, interactive tools are rarely used (Perkel, 2018) (e.g. https://www.elsevier.com/authors/tools-andresources/data-visualization), (https://aasnova.org/2021/02/26/ aas-publishing-news-interactive-figures), and have hardly been applied in the context of biomolecular structural information. As a result, the reader of a scientific paper has to rely on the interpretation of the author of a flat image, or alternatively, retrieve the structure from $\mathrm{PDB}$ and redo the imaging in a process that might take hours or even days.
Pioneering efforts towards figure interactivity have been done by different groups and consortia. The Interactive $3 D$ Complements (I3DC) from Proteopedia (Hodis et al., 2008) was a step forward for the incorporation of interactive structure visualizations as journal figures. POLYVIEW-3D (Porollo and Meller, 2007) offered a way to create publication quality structure renders, as well as animated images (GIF) to be used as dynamic figures, and the POLYVIEW-MM (Porollo and Meller, 2010) extension included the possibility to upload molecular simulation data, generating a combination of interactive visualization with structural analysis. Web3Dmol (Shi et al., 2017) was designed to be both an interactive protein structure visualizer and a tool to share interactive representations, being the first one integrating $1 \mathrm{D}$ sequence, measurement tools and meta-information. More recently, "I see in 3D" (iCin3D) offered a powerful macromolecular visualizer and editor, able to synchronize the display of $3 \mathrm{D}$ structure, 2D interaction, 1D sequences and annotations (Wang et al., 2020). The tool is integrated in the NCBI portfolio of services, and directly connected to the Vector Alignment Search Tool (VAST) (Berger et al., 2018) and ClinVar (Landrum et al., 2018) genomic variations database. In a similar way, ProSAT+ (Stank et al., 2016) and 3DBionotes (Tabas-Madrid et al., 2016) integrate biological annotations and structural information of proteins from multiple sources. 3DBionotes includes mutations, proteinprotein interactions, post-translational modifications (Segura et al., 2017) and most recently also genomic variations (Segura et al., 2019) and SARS-CoV-2 related annotations (Macias et al., 2021). Although the main goal of these tools is to extract evidence relating sequence, structure and function using the connection with external databases annotations, they all also offer the possibility to save the representation in a public URL.

$3 \mathrm{dRS}$ wants to contribute to the effort started by these projects and create an interactive environment favoring a full exploitation of the structural information of macromolecules. It is created from our experience in discussing structural data between remote centers and from the problems we experience when reading flat structural pictures. 3dRS offers an easy and intuitive web-based Graphical User Interface (GUI), with a curated user experience, to generate and share interactive macromolecular 3D representations. 3dRS can render more than one structure at the same time. Dynamic representations from $\mathrm{MD}$ trajectories can be incorporated thanks to the integrated MDsrv module. The generated scene can be shared with a permanent link, contributing to the FAIR guiding principles for data management (Wilkinson et al., 2016). Besides, the shared representations can be further modified and/or expanded, using a fork process, following the procedure used by the GitHub software development and version control repository. The 3dRS server has a very intuitive use, no registration is required and is freely available at https://mmb.irbbarcelona.org/3dRS.

\section{METHODS}

The server is divided in two main blocks: the front-end, embodied by the online accessible web server; and the back-end, where all 


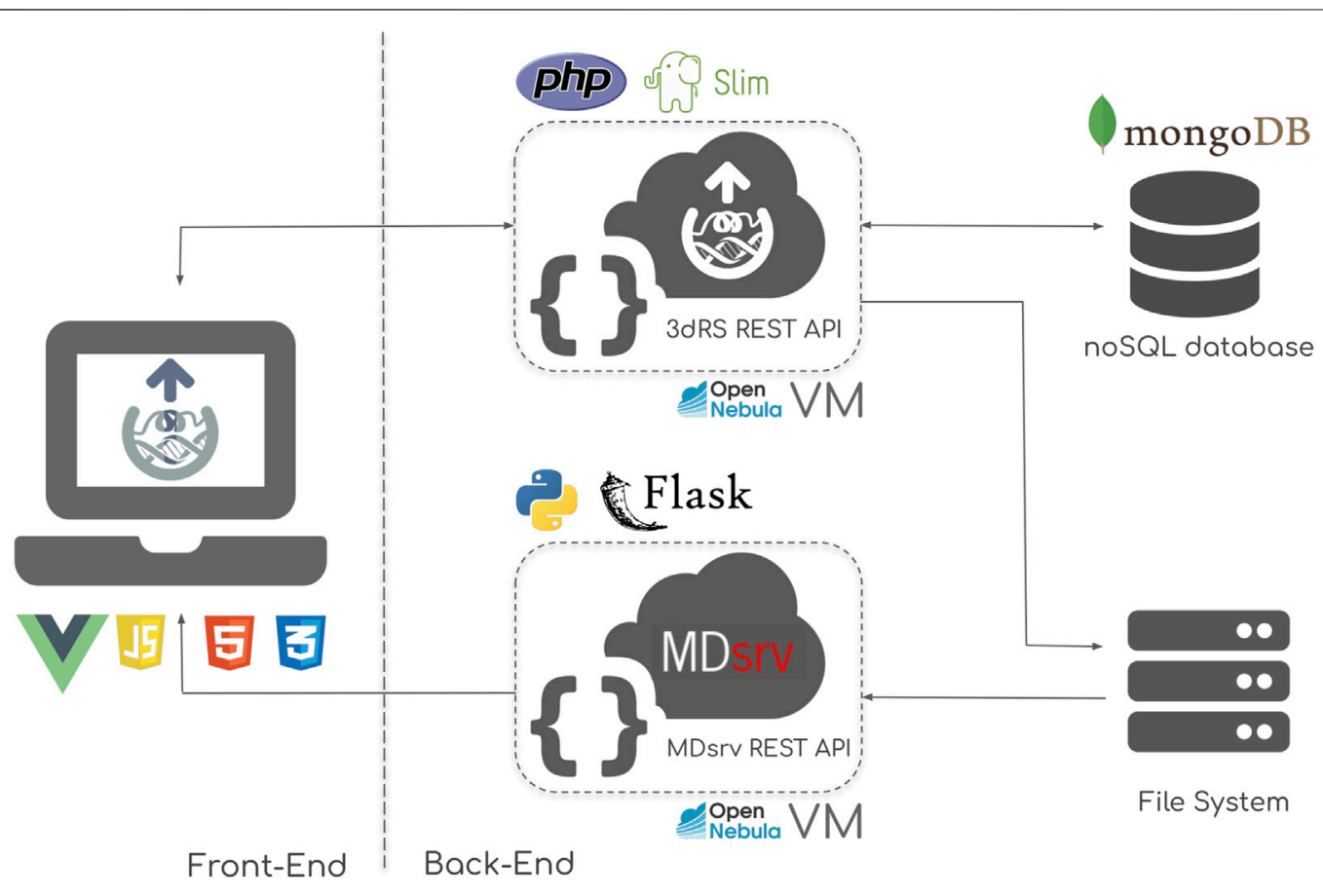

FIGURE 1 | 3D Representation Sharing infrastructure scheme, composed of two main sections: the front-end, powered by a combination of VueJS, HTML5 and CSS3; and the back-end, powered by a combination of PHP-Slim REST API, MDsrv MD trajectory streaming server, and mongoDB NoSQL database. The back-end tools are implemented in Virtual Machines (VM) deployed in an OpenNebula cloud infrastructure.

data, including the representation, structures and trajectories are stored and queried through REST APIs (Figure 1).

\section{Front-End}

The front-end implements the web-based Graphical User Interface (GUI) offered to the end user (Figure 1, left). This section includes the central part of the tool, i.e. the macromolecular structure representation routines, powered by the NGL viewer. VueJS, an open-source JavaScript framework is used for the central unit of the GUI. HTML5 and CSS3 technologies accompanying NGL and VueJS are used to polish up the final design. The result is a seamless user experience, mimicking that of a native, standalone program, without page reloads or saving buttons.

\section{Back-End}

The back-end of the infrastructure is divided into four main parts (Figure 1, right):

- 3dRS REST API: the core programmatic interface of the platform, written using the PHP Slim micro framework. This API is the responsible for communicating the web server (user actions) with the internal database and file system.

- MDsrv REST API: in charge of streaming MD trajectories data from the file system to the web-based interface. Written in Python Flask micro framework.

- mongoDB database: is the heart of the API, where all the representations created and structures uploaded by the end users are efficiently stored.

- File system: used for storing uploaded MD trajectories.
All the important information is automatically saved to the database using the $3 \mathrm{dRS}$ REST API, in a transparent manner.

\section{MODES}

3dRS offers an easy, web-based 3D macromolecular representation generator that can be then shared and reproduced with just a URL. Thus, the tool is divided in these two main working modes: edition and shared. The edition mode is used to add representations to the molecule(s). Such representations can be placed by selecting molecular regions and applying NGL drawing styles. Additional information such as measures (distances, angles) or labels can also be included. Once the final representation is ready, it can be shared through a permanent link. The scene is shared as it is shown in the editor, keeping not just the molecular representations, but also the orientation, zoom, background color, measures, and labels (if added). The permanent link opens the shared mode, which allows interactive exploration of the scene, hiding the representation menus. If the author of the scene has enabled forking, the project can be cloned, opening the edition mode again and recovering all the representations, which allows an easy creation of new scenes by just modifications on top of already existing projects.

\section{Edition Mode}

$3 \mathrm{dRS}$ edition mode is automatically opened after uploading one or more structures. It allows the creation of new representations 


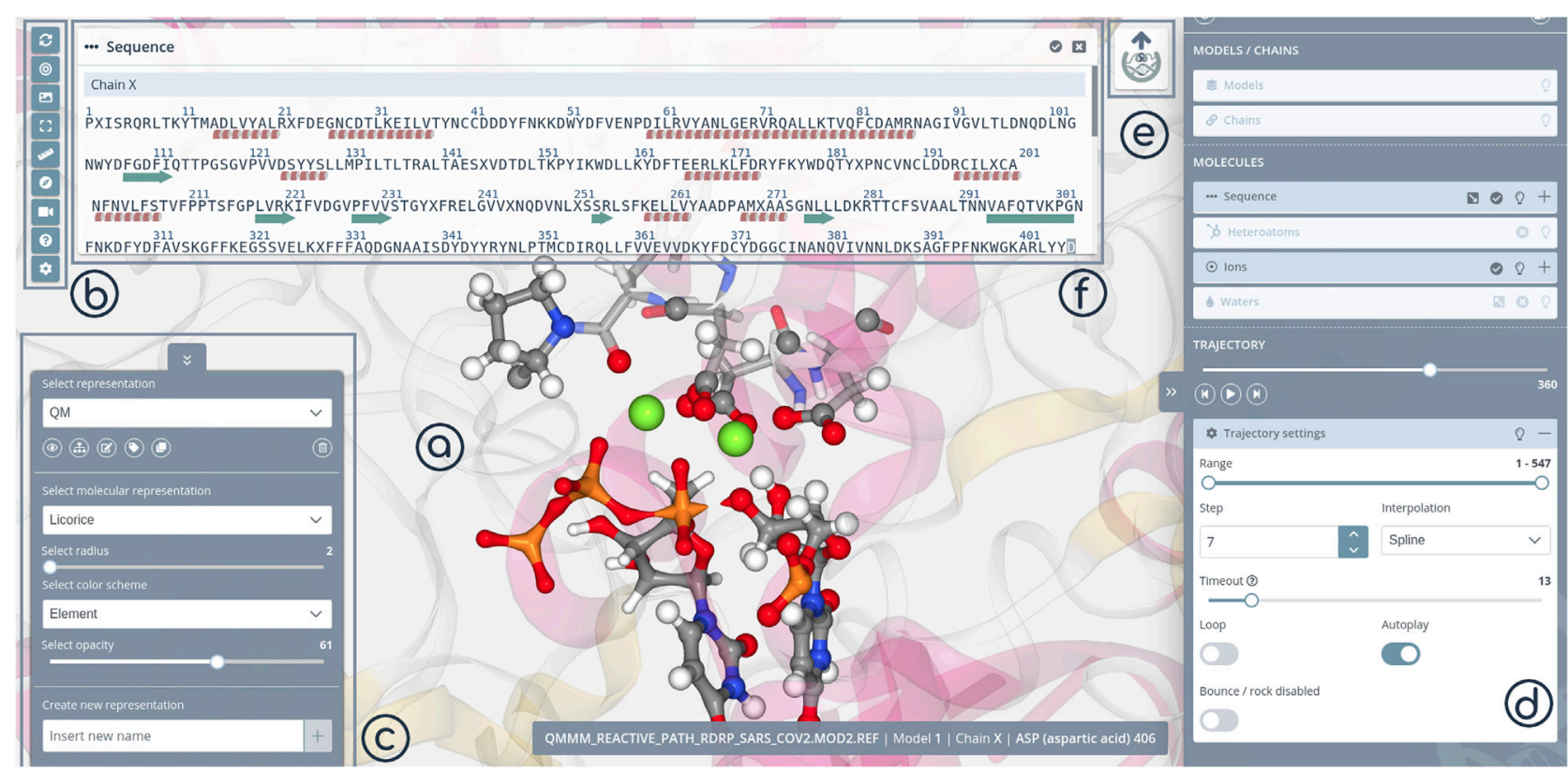

FIGURE 2 | 3dRS Edition Mode panels. (A) Stage panel; (B) Tools; (C) Representations; (D) Selections; (E) Sharing; (F) 1D Sequence.

with a live and interactive view of the structure(s) and the changes made in the central panel (Stage, Figure 2A). Visualization tools and project settings are accessible from a sidebar (Tools, Figure 2B). Available tools and settings vary depending on the working mode (edition/shared). New representations are added from a specific menu (Select Representation, Figure 2C). New representations are always linked to selections on the molecule(s), which are generated with a different specific menu (Selections, Figure 2D). The final representation can be shared clicking at the 3dRS logo button (Ready to share, Figure 2E).

The selection of the macromolecular regions to apply different drawing styles in $3 \mathrm{dRS}$ is inspired by the Visual Molecular Dynamics (VMD) tool (Humphrey et al., 1996), and is particularly powerful. Residue/Nucleotide sequences are opened in a new modal window (Figure 2F), which is synchronized with the $3 \mathrm{D}$ representation and the selection panel (Figures 2A,D). Pointing the mouse on a determined residue in the sequence window will highlight this residue in the representation and in the selection panel, and the other way around. Clicking on this residue will add it into the current selection. Segments of the sequence can be easily selected. To increase the selection power, a custom/manual selector is added, for advanced users to be able to use NGL syntax to generate complex selections.

\section{Shared Mode}

$3 \mathrm{dRS}$ shared mode is opened by default when using $3 \mathrm{dRS}$ sharing links. It presents an interactive figure, a representation of a macromolecular $3 \mathrm{D}$ structure that can be explored in $3 \mathrm{D}$, with the possibility to rotate, translate, zoom in/out and identify the atoms/residues/chains by just hovering on top of the particular molecular regions. Stage (Figure 3A) and Tools/Settings
(Figure 3B) panels are maintained, with the tools bar offering different features in this case (e.g. screenshot, embed representation, QR code). Edition panels (Representations, Selections) are substituted here by the figure caption (Figure 3C), which works exactly as the captions in scientific journals, with the advantages of the web, adding rich text and including external links. Finally, the sharing logo button has been substituted by the Forking button (Figure 3D), giving the possibility to clone the project to either extend or modify it. This feature is only available if the original author of the representation has enabled forking on the project. In case of sharing a structure and a trajectory, an easy player is shown on the top of the screen (Figure 3E). This player offers the ability of controlling the given trajectory in a simple way (play/pause, move frame by frame back and forward and jump to a concrete frame through the slider bar).

\section{RESULTS}

$3 \mathrm{dRS}$ web platform allows the generation of shareable interactive $3 \mathrm{D}$ representations from a very easy and intuitive interface. It can load structures directly from the PDB databank (PDB code) or from disk (PDB file). Multiple structures can be submitted at once, with the possibility to superpose them once loaded. MD trajectory data can be inserted for each of the structures in the scene, with an efficient streaming management thanks to the integrated MDsrv tool. 3dRS is compatible with PDB and GRO input structure files formats, with a size limit of $50 \mathrm{MB}$. For the trajectory data, XTC, DCD, TRR, BINPOS, and NETCDF formats are accepted, with a file size limit of $500 \mathrm{MB}$. Measures (distances, angles) and labels can be easily inserted in the scene. The whole 


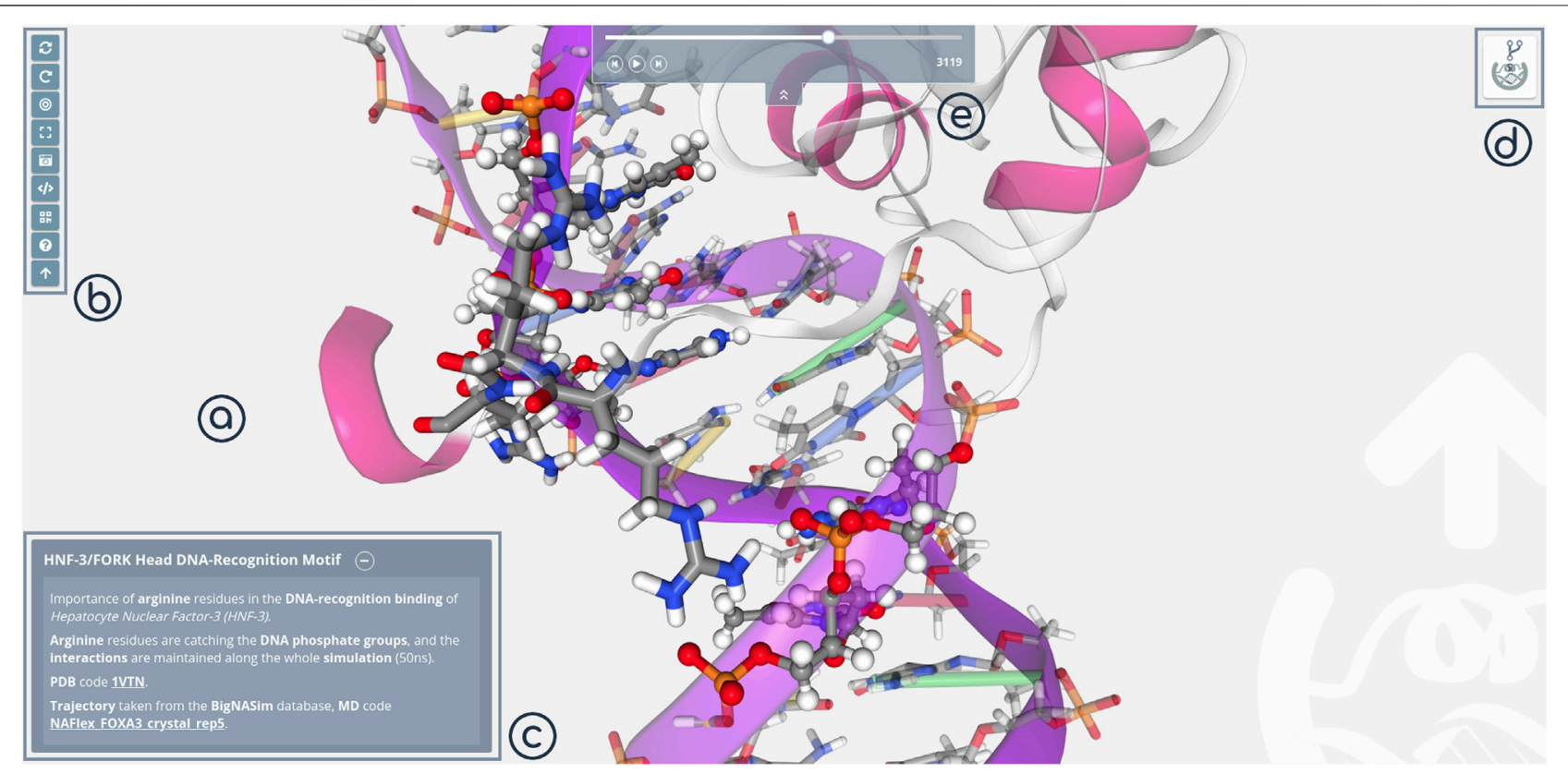

FIGURE 3 | 3dRS Shared Mode panels. (A) Stage panel; (B) Tools; (C) Caption; (D) Forking; (E) Player.

representation can be shared with just a permanent URL link, with the possibility to extend or modify the representation, forking the project.

3dRS follows FAIR data guiding principles (Wilkinson et al., 2016). Representations have a globally unique and persistent identifier (permanent link). They are accessible using a standardized communications protocol (web browser). Metadata included in the representation can be used to determine the structure provenance (PDB, local file) and to further reproduce and extend the generated scene (fork). FAIR principles have been also applied in the software development process (Lamprecht et al., 2019). Accordingly, source code is available from GitHub repositories. The tool is registered in the bio.tools bioinformatics and Life Sciences registry (Ison et al., 2016) (https://bio.tools/3drs). Extended documentation is available from ReadTheDocs, a domain-level community standard. All the software is licensed under the Apache 2.0 license.

Examples of what $3 \mathrm{dRS}$ could offer to the scientific community can be found in the project gallery (https://mmb. irbbarcelona.org/3dRS/gallery). Three of these examples have been selected to demonstrate the power of the tool and are described in the following sections.

\section{Example 1-SARS-CoV-2 Receptor Binding Domain (RBD) Variants}

Scientific groups around the world are devoting their efforts in the study of the SARS-CoV-2 and particularly in the Spike protein, a trimeric protein crucial in the virus infection and target for most of the current vaccines. Hundreds of studies directed to this protein are now available, a big part focusing on the different variants lately discovered and their impact in infectivity and vaccination resistance. Representations of the trimeric protein with the variants and known associated mutations/deletions occurring in the same lineage allow a quick identification of the location of the variants with respect to the main spike domains, with particular interest on the Receptor Binding Domain (RBD) and N-Terminal Domain (NTD), both known to contain epitopes and thus targeted regions of antibodies. $3 \mathrm{dRS}$ was used to generate a collection of interactive SARS-CoV-2 representations, from the complete Spike protein to the insights of the recognition interface between $\mathrm{RBD}$ and the human Angiotensin Converting Enzyme 2 (hACE2) (Figure 4).

Figure 4A (https://mmb.irbbarcelona.org/3dRS/s/c1tOez) and Figure 4B (https://mmb.irbbarcelona.org/3dRS/s/ hfS2rW) show the trimeric Spike protein representation, with the most important RBD variants highlighted in red, associated mutations/deletions in yellow, and epitopes in green. RBD region is displayed in blue color, whereas the NTD domain is displayed in red color. Figure 4B is showing a particular Spike conformation with one RBD region in "up" position, already detached from the main trimer and prepared to recognize the human cell. Figure $4 \mathrm{C}$ (https://mmb. irbbarcelona.org/3dRS/s/lQ6f21) and Figure 4D (https:// mmb.irbbarcelona.org/3dRS/s/hJxjZv) show a particular monomeric structure from the Spike trimer with the RBD in "up" position, attached to the antibody P5A-1B9 Fab (PDB code 7CZX) (Figure 4C) and with the NTD domain attached to the antibody 1-87 (PDB code 7L2D) (Figure 4D). Finally, Figure 4E (https://mmb.irbbarcelona.org/3dRS/s/JcshuF) and Figure 4F (https://mmb.irbbarcelona.org/3dRS/s/ 


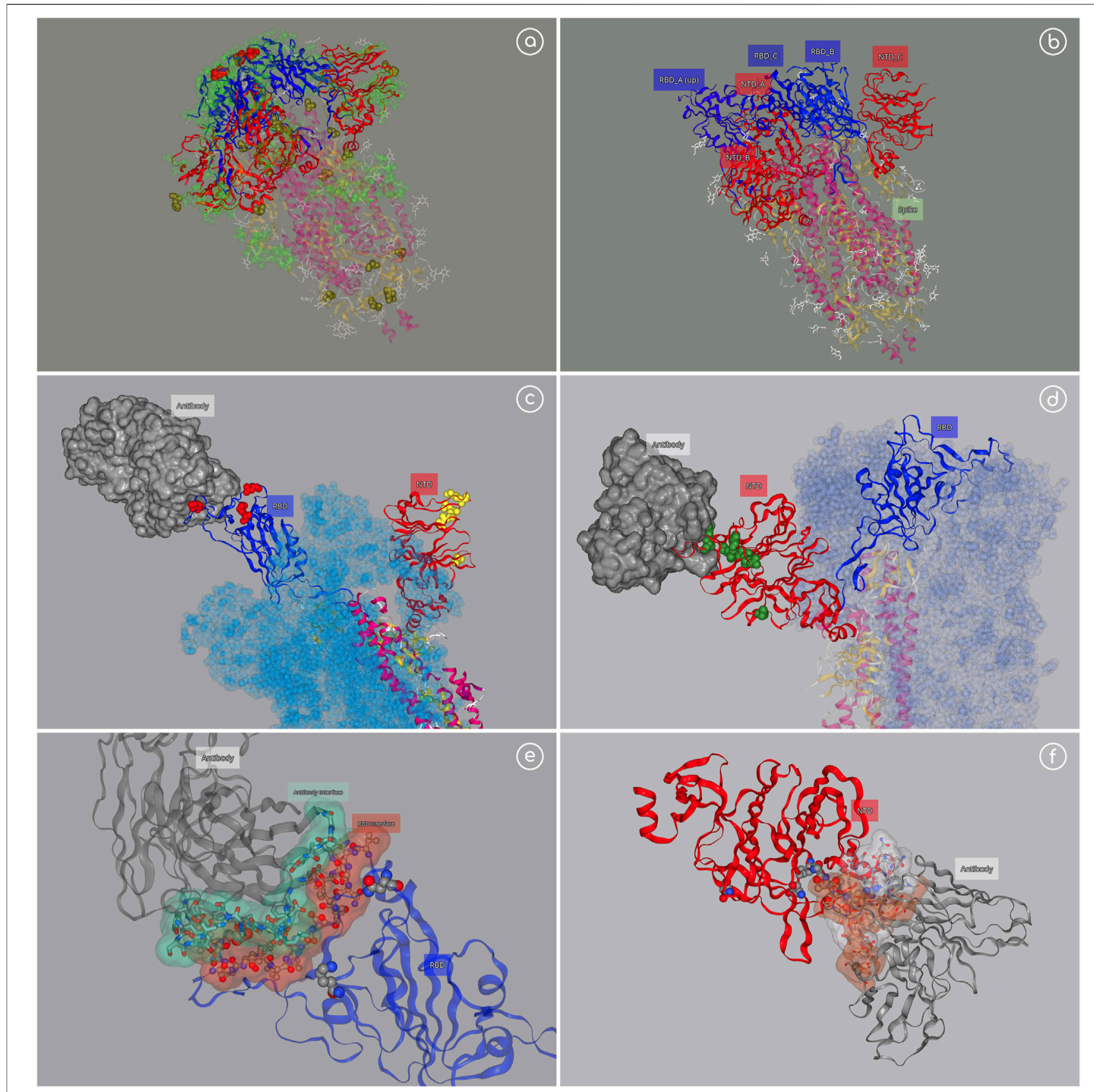

FIGURE 4 | SARS-CoV-2 example. (A) Spike trimeric protein; https://mmb.irbbarcelona.org/3dRS/s/c1tOez; (B) Spike with one RDB domain in "up" position; https://mmb.irbbarcelona.org/3dRS/s/hfS2rW; (C) Spike (monomer) with P5A-1B9 Fab antibody attached to the RBD domain; https://mmb.irbbarcelona.org/3dRS/s/ IQ6f21; (D) Spike (monomer) with 1-87 antibody attached to the NTD domain; https://mmb.irbbarcelona.org/3dRS/s/hJxiZv (E) Insight of RBD-Antibody interface; https://mmb.irbbarcelona.org/3dRS/s/JcshuF (F) Insight of NTD-Antibody interface; https://mmb.irbbarcelona.org/3dRS/s/YiGhxv.

YiGhxv) represent an insight on the interaction regions between the RBD/NTD and the antibodies.

\section{Example 2-SARS-CoV-2 RNA-dependent RNA Polymerase}

SARS-CoV-2 RNA-dependent RNA polymerase (RdRp) is the main protein for virus replication after binding to non-structural proteins (nsp) cofactors nsp7 and nsp8 to form an active complex (Kirchdoerfer and Ward, 2019). Recent studies in the group (Aranda and Orozco, 2020) have shown that the RdRp reaction process is able to select the entering nucleotide and to catalyze its addition to a nascent RNA, following a mechanism that is similar to that of bacterial or eukaryotic polymerases with the transferred phosphate being stabilized by two $\mathrm{Mg}^{2+}$ ions coordinated by acidic residues of the catalytic site, while the 

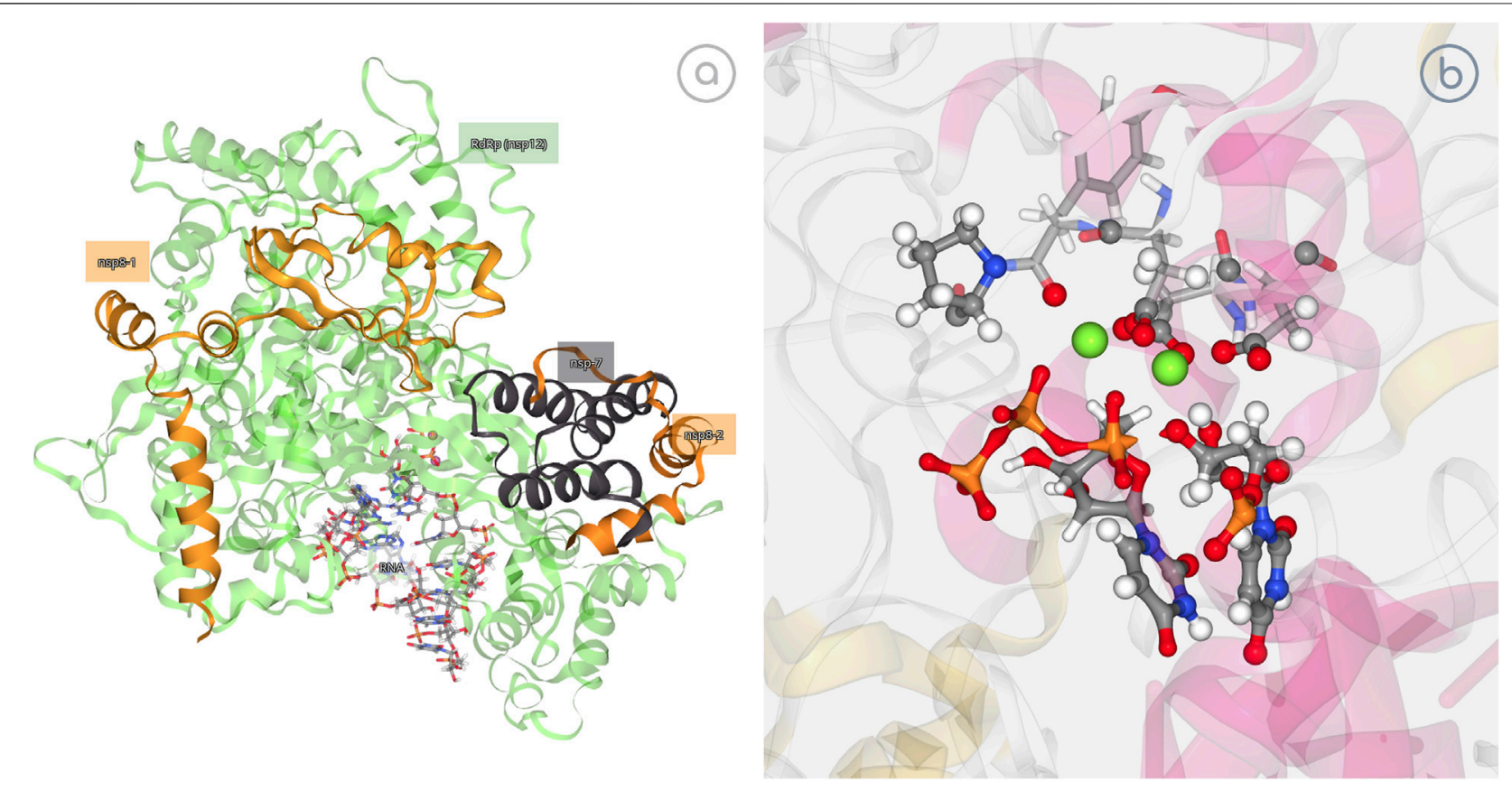

FIGURE 5 | SARS-CoV-2 RNA-Dependent RNA Polymerase (RdRp) study. (A) Interactive representation of RdRp bound to its essential co-factors nsp7 and nsp8; https://mmb.irbbarcelona.org/3dRS/s/7Rn2Ka; (B) Exploring the ability of RdRp to incorporate a natural triphosphate into a nascent RNA; https://mmb.irbbarcelona. org/3dRS/s/TKf8yA.

phosphates of the incoming nucleotide being stabilized by a network of basic residues (Aranda and Orozco, 2020). In this example, the possibility of $3 \mathrm{dRS}$ to also represent breaking or formation of bonds as studied by QM/MM methods is showcased. 3dRS was used to generate first an interactive figure of the SARS-CoV-2 RdRp in which the attached cofactors and nucleotides are highlighted, introducing the $3 \mathrm{D}$ structure. Then, the process of incorporation of a natural triphosphate into a nascent RNA, exemplified by the breaking of a P1-O3P bond and the following formation of the P1-O3' bond, is represented in an interactive and dynamic figure (Figure 5).

Figure 5A (https://mmb.irbbarcelona.org/3dRS/s/7Rn2Ka) and Figure 5B (https://mmb.irbbarcelona.org/3dRS/s/TKf8yA) show the SARS-CoV-2 RdRp representations. Figure $\mathbf{5 A}$ is introducing the SARS-CoV-2 RNA-Dependent RNA Polymerase bound to its essential co-factors nsp7 and nsp8, whereas Figure $\mathbf{5 B}$ is representing the reaction process involving a bond breaking and a new bond formation.

\section{Example 3-Collective Variables From Coarse-Grained Conformational Transitions}

Large protein conformational changes are difficult to study experimentally and often not accessible for accurate atomistic models, forcing the use of Coarse-Grained Molecular Dynamics algorithms (Orozco et al., 2011). As an example, GOdMD method (Sfriso et al., 2013) can be used to extract relevant biophysical information to rationalize the protein motion, including a robust set of collective variables (CVs) capturing the conformational change. These CVs can then be used in more expensive and accurate calculations to refine the representation of the conformational transition. $3 \mathrm{dRS}$ allows the interactive and dynamic representation of some conformational transitions, highlighting the captured collective variables and showing the transition from trajectory data (Figure 6).

Figure 6A (https://mmb.irbbarcelona.org/3dRS/s/nDM5sO) and Figure 6B (https://mmb.irbbarcelona.org/3dRS/s/ QGAgYO) show the conformational transitions computed with the GOdMD package for the D-ribose binding protein (Figure 6A) and the Calcium Saturated Cardiac Troponin C (Figure 6B), highlighting the collective variables with red dots. Distance between the atoms contained in the collective variables is shown as a reference.

\section{DISCUSSION}

3D Representation Sharing platform is a demonstration of the technical revolution in the biomolecular simulation field. Illustrations of biomolecular structures in scientific journals and conference proceeding linked for years to static, $2 \mathrm{D}$ figures, can be finally changed for living, interactive, 3dimensional representations. This change, considered futuristic just 10 years ago, is now possible thanks to the fast evolution of the technology, with high-speed data transfer, greater connection bandwidths, efficient data storage tools as offered by NoSQL databases, and biomolecular visualizers able to efficiently run in common 


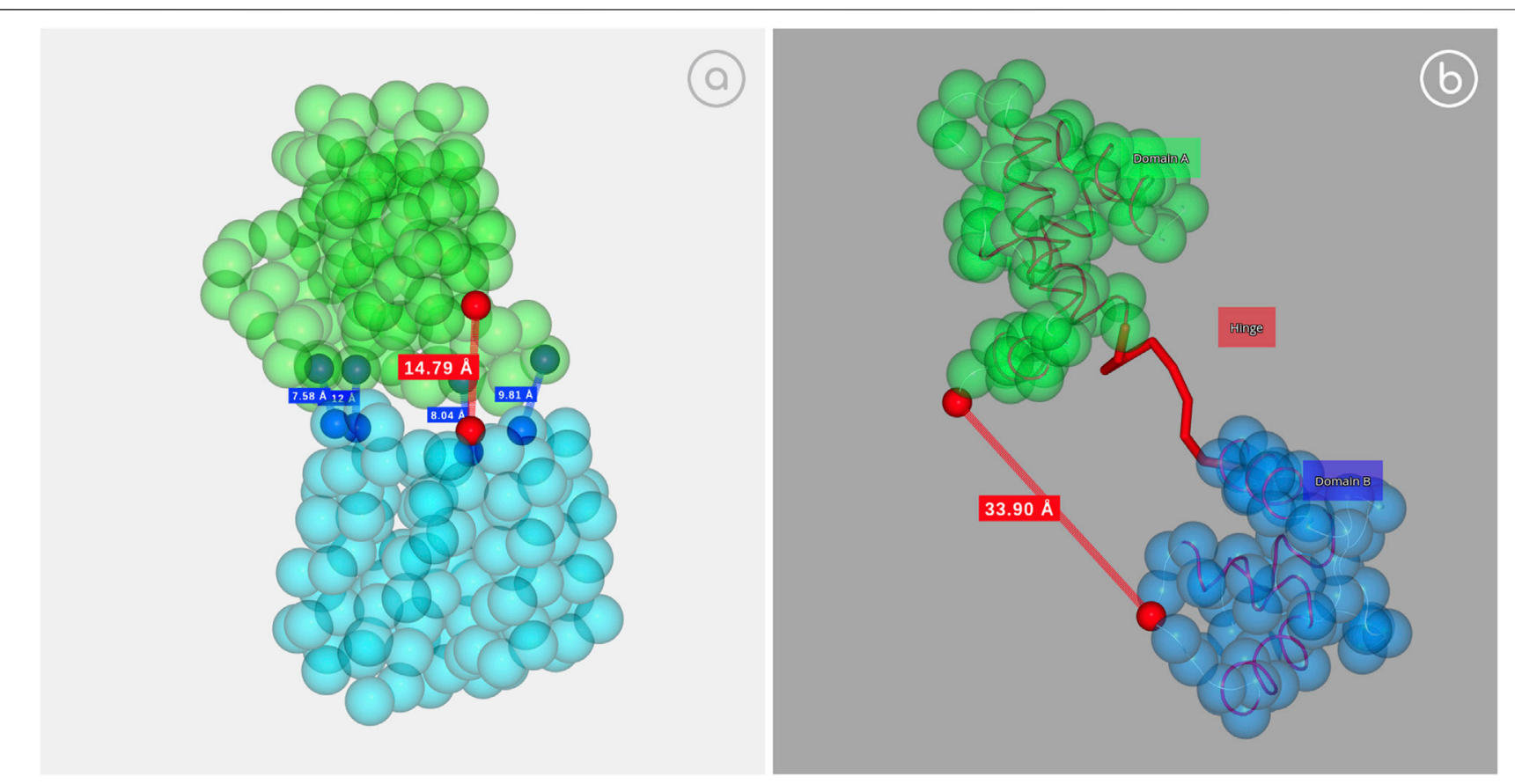

FIGURE 6 | Collective variables example. (A) D-Ribose binding protein; https://mmb.irbbarcelona.org/3dRS/s/nDM5sO; (B) Calcium Saturated Cardiac Troponin C; https://mmb.irbbarcelona.org/3dRS/s/QGAgYO.

web browsers and multiple platforms and take advantage of new generation GPUs.

3dRS emerge from our experience in discussing structural models in the context of large international consortia, such as BioExcel Centre of Excellence (BioExcel CoE), ELIXIR, or Human Brain Project (HBP), where standard imaging techniques are not able to provide the desired level of interactivity between the image and the groups, based in different countries. Although an image of the structure with measurements and labels gives a first glance of these important features, it is inevitable for a scientist to wonder what is happening just behind or around the interaction, unconsciously thinking in three dimensions. And this reaction is even stronger if the picture is presenting information coming from an MD flexibility analysis, where specific molecular regions are changing depending on the frame analyzed. Interactive figures created with $3 \mathrm{dRS}$ are making this possible.

$3 \mathrm{dRS}$ is to our knowledge, the only available tool making the sharing of interactive and dynamic $3 \mathrm{D} / 4 \mathrm{D}$ custom representations possible. It keeps the process as simple as possible, easing the representation generation to reach a broader scientific community, including that with a limited knowledge on recent technologies. Generated representations are not linked to any kind of analysis or external databases as found in $\mathrm{iCn} 3 \mathrm{D}$ or 3DBionotes platforms. The scene is shown and shared as the author conceived it, facilitating the author to highlight the details of importance in the global structure, hiding or giving transparency to less important regions of the structure, making it ideal for scientific discussion.

Quite unique of $3 \mathrm{dRS}$, and also a consequence of our involvement in international consortia massively using atomistic simulations is the possibility to share MD trajectory data. Although limited in size by the web technology, the possibility to include this dynamic data is hugely increasing the value of the final representation. A small number of webbased servers can present 3D-interactive MD trajectories so far, like BigNASim (Hospital et al., 2016), or GPCRmd databases (Rodríguez-Espigares et al., 2020) (the latter relying on MDsrv and NGL tools as 3dRS), but still most dynamic information is kept and unshared in local computers. The presented platforms are designed to specifically work with local MD databases. $3 \mathrm{dRS}$ is opening these technologies so that the whole community can build and share dynamic figures representing MD trajectories.

$3 \mathrm{dRS}$ software is open source (Apache2 license) and can be found in: https://github.com/gbayarri?tab=repositories\&q=3drs. Documentation is written using ReadTheDocs technology, and is available from this link: https://3drs-documentation.readthedocs. io/en/latest/. The software is platform independent. It is a webbased tool, working in the most common Internet browser tools, although Google Chrome browser is recommended. The frontend is written as a Single Page Application (SPA) using VueJS, HTML5 and CSS3 technologies. The back-end is written with the PHP-Slim framework, with the MongoDB PHP Driver connecting to the underlying database, and the MDsrv server streaming MD data.

\section{DATA AVAILABILITY STATEMENT}

The original contributions presented in the study are publicly available. This data can be found here: https:/github.com/ gbayarri?tab=repositories $\& \mathrm{q}=3 \mathrm{drs}$. 


\section{AUTHOR CONTRIBUTIONS}

GB and AH contributed to the conception and design of the study. GB implemented the database, backend and frontend code. MO supervised the overall research. $\mathrm{GB}, \mathrm{AH}$ and $\mathrm{MO}$ wrote the manuscript. All authors contributed to manuscript revision, read, and approved the submitted version.

\section{FUNDING}

This work was funded by the Spanish Ministry of Science (Grant No. RTI 2018-096704-B-100), the Catalan SGR, the Instituto Nacional de Bioinformática; European Union's Horizon 2020 research and innovation program (BioExcel-2 project, 823,830),

\section{REFERENCES}

Andrei, R., Callieri, M., Zini, M., Loni, T., Maraziti, G., Pan, M., et al. (2012). Intuitive Representation of Surface Properties of Biomolecules Using BioBlender. BMC Bioinformatics 13 (Suppl. 4), S16. doi:10.1186/1471-210513-s4-s16

Aranda, J., and Orozco, M. (2020). RNA-dependent RNA Polymerase from SARSCoV-2 Mechanism of Reaction and Inhibition by Remdesivir. bioRxiv. 2020. doi:10.1101/2020.06.21.163592

Bekker, G. J., Nakamura, H., and Kinjo, A. R. (2016). Molmil: a Molecular Viewer for the PDB and beyond. J. Cheminform 8 (1), 42. doi:10.1186/s13321-0160155-1

Berger, D. R., Seung, H. S., and Lichtman, J. W. (2018). VAST (Volume Annotation and Segmentation Tool): Efficient Manual and Semi-automatic Labeling of Large 3D Image Stacks. Front. Neural Circuits 12, 88. doi:10.3389/ fncir.2018.00088

Berman, H. M., Westbrook, J., Feng, Z., Gilliland, G., Bhat, T. N., Weissig, H., et al. (2000). The Protein Data Bank. Nucleic Acids Res. 28 (1), 235-242. doi:10.1093/ nar/28.1.235

Carrillo-Tripp, M., Alvarez-Rivera, L., Lara-Ramírez, O. I., Becerra-Toledo, F. J., Vega-Ramírez, A., Quijas-Valades, E., et al. (2018). HTMoL: Full-Stack Solution for Remote Access, Visualization, and Analysis of Molecular Dynamics Trajectory Data. J. Comput. Aided Mol. Des. 32 (8), 869-876. doi:10.1007/s10822-018-0141-y

Hildebrand, P. W., Rose, A. S., and Tiemann, J. K. S. (2019). Bringing Molecular Dynamics Simulation Data into View. Trends Biochem. Sci. 44 (11), 902-913. doi:10.1016/j.tibs.2019.06.004

Hodis, E., Prilusky, J., Martz, E., Silman, I., Moult, J., and Sussman, J. L. (2008). Proteopedia - a Scientific 'wiki' Bridging the Rift between 3D Structure and Function of Biomacromolecules. Genome Biol. 9 (8), R121. doi:10.1186/gb2008-9-8-r121

Hospital, A., Goñi, J. R., Orozco, M., and Gelpí, J. L. (2015). Molecular Dynamics Simulations: Advances and Applications. Adv. Appl. Bioinform Chem. 8, 37-47. doi:10.2147/AABC.S70333

Hospital, A., Andrio, P., Cugnasco, C., Codo, L., Becerra, Y., Dans, P. D., et al. (2016). BIGNASim: a NoSQL Database Structure and Analysis portal for Nucleic Acids Simulation Data. Nucleic Acids Res. 44 (D1), D272-D278. doi:10.1093/nar/gkv1301

Humphrey, W., Dalke, A., and Schulten, K. (1996). VMD: Visual Molecular Dynamics. J. Mol. Graphics 14 (1), 33-38. doi:10.1016/0263-7855(96)00018-5

Ison, J., Rapacki, K., Ménager, H., Kalaš, M., Rydza, E., Chmura, P., et al. (2016). Tools and Data Services Registry: a Community Effort to Document Bioinformatics Resources. Nucleic Acids Res. 44 (D1), D38-D47. doi:10.1093/nar/gkv1116

Johnson, G. T., Autin, L., Goodsell, D. S., Sanner, M. F., and Olson, A. J. (2011). ePMV Embeds Molecular Modeling into Professional Animation Software Environments. Structure 19 (3), 293-303. doi:10.1016/j.str.2010.12.023
Biomolecular and Bioinformatics Resources Platform (Grant No. ISCIII PT 13/0001/0030) cofunded by the Fondo Europeo de Desarrollo Regional (FEDER) and the EU Human Brain Project Flagship as well as the MINECO Severo Ochoa Award of Excellence (Government of Spain). MO is an Icrea Academy Researcher.

\section{ACKNOWLEDGMENTS}

The authors warmly thank Josep Lluís Gelpí (BSC) for his useful discussions, Juan Aranda for sharing and helping with the QM/MM trajectory example, Francesco Colizzi and Federica Battistini for generous sharing of MD trajectories for the gallery.

Kirchdoerfer, R. N., and Ward, A. B. (2019). Structure of the SARS-CoV Nsp12 Polymerase Bound to Nsp7 and Nsp8 Co-factors. Nat. Commun. 10 (1), 2342. doi:10.1038/s41467-019-10280-3

Lamprecht, A.-L., Garcia, L., Kuzak, M., Martinez, C., Arcila, R., Martin Del Pico, E., et al. (2019). Towards FAIR Principles for Research Software. Data Science, IOS Press.

Landrum, M. J., Lee, J. M., Benson, M., Brown, G. R., Chao, C., Chitipiralla, S., et al. (2018). ClinVar: Improving Access to Variant Interpretations and Supporting Evidence. Nucleic Acids Res. 46 (D1), D1062-D1067. doi:10.1093/nar/gkx1153

Macias, J. R., Sanchez-Garcia, R., Conesa, P., Ramirez-Aportela, E., Gonzalez, M. M., Wert-Carvajal, C., et al. (2021). 3DBionotes COVID-19 Edition. Bioinformatics. doi:10.1093/bioinformatics/btab397

McGibbon, R. T., Beauchamp, K. A., Harrigan, M. P., Klein, C., Swails, J. M., Hernández, C. X., et al. (2015). MDTraj: A Modern Open Library for the Analysis of Molecular Dynamics Trajectories. Biophysical J. 109 (8), 1528-1532. doi:10.1016/j.bpj.2015.08.015

Meyer, T., D’Abramo, M., Hospital, A., Rueda, M., Ferrer-Costa, C., Pérez, A., et al. (2010). MoDEL (Molecular Dynamics Extended Library): a Database of Atomistic Molecular Dynamics Trajectories. Structure 18 (11), 1399-1409. doi:10.1016/j.str.2010.07.013

Michaud-Agrawal, N., Denning, E. J., Woolf, T. B., and Beckstein, O. (2011). MDAnalysis: A Toolkit for the Analysis of Molecular Dynamics Simulations. J. Comput. Chem. 32 (10), 2319-2327. doi:10.1002/jcc.21787

Orozco, M., Orellana, L., Hospital, A., Naganathan, A. N., Emperador, A., Carrillo, O., et al. (2011). Coarse-grained Representation of Protein Flexibility. Foundations, Successes, and Shortcomings. Adv. Protein Chem. Struct. Biol. 85, 183-215. doi:10.1016/b978-0-12-386485-7.00005-3

Perkel, J. M. (2018). Data Visualization Tools Drive Interactivity and Reproducibility in Online Publishing. Nature 554 (7690), 133-134. doi:10.1038/d41586-018-01322-9

Perutz, M. F., Rossmann, M. G., Cullis, A. F., Muirhead, H., Will, G., and North, A. C. T. (1960). Structure of Hæmoglobin: A Three-Dimensional Fourier Synthesis at 5.5- $\AA$. Resolution, Obtained by X-Ray Analysis. Nature 185 (4711), 416-422. doi:10.1038/185416a0

Porollo, A., and Meller, J. (2010). POLYVIEW-MM: Web-Based Platform for Animation and Analysis of Molecular Simulations. Nucleic Acids Res. 38, W662-W666. doi:10.1093/nar/gkq445

Porollo, A., and Meller, J. (2007). Versatile Annotation and Publication Quality Visualization of Protein Complexes Using POLYVIEW-3D. BMC Bioinformatics 8, 316. doi:10.1186/1471-2105-8-316

Rego, N., and Koes, D. (2015). 3Dmol.js: Molecular Visualization with WebGL. Bioinformatics 31 (8), 1322-1324. doi:10.1093/bioinformatics/ btu 829

Rodríguez-Espigares, I., Torrens-Fontanals, M., Tiemann, J. K. S., Aranda-García, D., Ramírez-Anguita, J. M., Stepniewski, T. M., et al. (2020). GPCRmd Uncovers the Dynamics of the 3D-GPCRome. Nat. Methods 17 (8), 777-787. doi:10.1038/s41592-020-0884-y 
Rose, A. S., Bradley, A. R., Valasatava, Y., Duarte, J. M., Prlic, A., and Rose, P. W. (2018). NGL Viewer: Web-Based Molecular Graphics for Large Complexes. Bioinformatics 34, 3755-3758. doi:10.1093/bioinformatics/bty419

Segura, J., Sanchez-Garcia, R., Martinez, M., Cuenca-Alba, J., Tabas-Madrid, D., Sorzano, C. O. S., et al. (2017). 3DBIONOTES v2.0: a Web Server for the Automatic Annotation of Macromolecular Structures. Bioinformatics 33 (22), 3655-3657. doi:10.1093/bioinformatics/btx483

Segura, J., Sanchez-Garcia, R., Sorzano, C. O. S., and Carazo, J. M. (2019). 3DBIONOTES v3.0: Crossing Molecular and Structural Biology Data with Genomic Variations. Bioinformatics 35 (18), 3512-3513. doi:10.1093/ bioinformatics/btz118

Sehnal, D., Deshpande, M., Vařeková, R. S., Mir, S., Berka, K., Midlik, A., et al. (2017). LiteMol Suite: Interactive Web-Based Visualization of Large-Scale Macromolecular Structure Data. Nat. Methods 14 (12), 1121-1122. doi:10.1038/nmeth.4499

Sehnal, D., Bittrich, S., Deshpande, M., Vařeková, R. S., Berka, K., Bazgier, V., et al. (2021). $\mathrm{Mol}^{\star}$ Viewer: Modern Web App for 3D Visualization and Analysis of Large Biomolecular Structures. Nucleic Acids Res.

Senior, A. W., Evans, R., Jumper, J., Kirkpatrick, J., Sifre, L., Green, T., et al. (2020). Improved Protein Structure Prediction Using Potentials from Deep Learning. Nature 577 (7792), 706-710. doi:10.1038/s41586-019-1923-7

Sfriso, P., Hospital, A., Emperador, A., and Orozco, M. (2013). Exploration of Conformational Transition Pathways from Coarse-Grained Simulations. Bioinformatics 29 (16), 1980-1986. doi:10.1093/bioinformatics/btt324

Shi, M., Gao, J., and Zhang, M. Q. (2017). Web3DMol: Interactive Protein Structure Visualization Based on WebGL. Nucleic Acids Res. 45 (W1), W523-W527. doi:10.1093/nar/gkx383

Stank, A., Richter, S., and Wade, R. C. (2016). ProSAT+: Visualizing Sequence Annotations on 3D Structure. Protein Eng. Des. Selection 29 (8), 281-284. doi:10.1093/protein/gzw021

Tabas-Madrid, D., Segura, J., Sanchez-Garcia, R., Cuenca-Alba, J., Sorzano, C. O. S., and Carazo, J. M. (2016). 3DBIONOTES: A Unified, Enriched and Interactive View of Macromolecular Information. J. Struct. Biol. 194 (2), 231-234. doi:10.1016/j.jsb.2016.02.007

Tiemann, J. K. S., Guixà-González, R., Hildebrand, P. W., and Rose, A. S. (2017). MDsrv: Viewing and Sharing Molecular Dynamics Simulations on the Web. Nat. Methods 14 (12), 1123-1124. doi:10.1038/nmeth.4497
Walters, W. P., and Wang, R. (2020). New Trends in Virtual Screening. J. Chem. Inf. Model. 60 (9), 4109-4111. doi:10.1021/acs.jcim.0c01009

Wang, J., Youkharibache, P., Zhang, D., Lanczycki, C. J., Geer, R. C., Madej, T., et al. (2020). iCn3D, a Web-Based 3D Viewer for Sharing 1D/2D/3D Representations of Biomolecular Structures. Bioinformatics 36 (1), 131-135. doi:10.1093/bioinformatics/btz502

Wieczór, M., Hospital, A., Bayarri, G., Czub, J., and Orozco, M. (2020). Molywood: Streamlining the Design and Rendering of Molecular Movies. Bioinformatics 36 (17), 4660-4661. doi:10.1093/bioinformatics/btaa584

Wilkinson, M. D., Dumontier, M., Aalbersberg, I. J., Appleton, G., Axton, M., Baak, A., et al. (2016). The FAIR Guiding Principles for Scientific Data Management and Stewardship. Sci. Data 3, 160018.

Zhu, L., Davari, M. D., and Li, W. (2021). Recent Advances in the Prediction of Protein Structural Classes: Feature Descriptors and Machine Learning Algorithms. Crystals 11 (4). doi:10.3390/cryst11040324

Zivanovic, S., Bayarri, G., Colizzi, F., Moreno, D., Gelpí, J. L., Soliva, R., et al. (2020). Bioactive Conformational Ensemble Server and Database. A Public Framework to Speed up In Silico Drug Discovery. J. Chem. Theor. Comput. 16 (10), 6586-6597. doi:10.1021/acs.jctc.0c00305

Conflict of Interest: The authors declare that the research was conducted in the absence of any commercial or financial relationships that could be construed as a potential conflict of interest.

Publisher's Note: All claims expressed in this article are solely those of the authors and do not necessarily represent those of their affiliated organizations, or those of the publisher, the editors and the reviewers. Any product that may be evaluated in this article, or claim that may be made by its manufacturer, is not guaranteed or endorsed by the publisher.

Copyright (c) 2021 Bayarri, Hospital and Orozco. This is an open-access article distributed under the terms of the Creative Commons Attribution License (CC BY). The use, distribution or reproduction in other forums is permitted, provided the original author(s) and the copyright owner(s) are credited and that the original publication in this journal is cited, in accordance with accepted academic practice. No use, distribution or reproduction is permitted which does not comply with these terms. 